\title{
Multifunctional decorative coatings based on cement-silicate binder
}

\author{
Arina Shaybadullina ${ }^{1, *}$, Grygory Yakovlev ${ }^{1}$, Valery Grakhov ${ }^{1}$, Irina Polyanskikh ${ }^{1}$, and \\ Yuliya Ginchitskaya ${ }^{1}$ \\ ${ }^{1}$ Kalashnikov Izhevsk State Technical University, Studencheskaya Str., 7, Izhevsk 426069, Russian \\ Federation
}

\begin{abstract}
A facade decorative coating has been developed that is based on sodium silicate and Portland cement and modified with a complex ultra- and nanodispersed admixture including titanium dioxide, expanded perlite sand, and multi-walled carbon nanotubes dispersion. The advantage of the produced coating is using Portland cement as a silicizer instead of the conventionally used zinc oxide. Adding ultra- and nanodispersed admixtures to the cement-silicate composition leads to the structural modification of the matrix of the binder composition along with the formation of a more durable coating (up to 4-5 times compared with the existing analogues) and the possibility of absorbing man-made electromagnetic radiation up to $38 \%$. Expanded pearlite sand in the silicate coating provides a relief surface when applied on the base. The cement-silicate composition for coating facades is shown to have the following physical and technical characteristics: the viscosity measured with Viscometer- 6 is $29 \mathrm{sec}$; the hydrogen index of the medium $\mathrm{pH}=12.33$; the film resistance to static effect of water is $8 \mathrm{hr}$; the paint consumption for a two-layer coating is $200-400 \mathrm{~g} / \mathrm{m}^{2}$; the adhesion (the cross-cut test) is 1 point; the conventional light-fastness is $4-5$ points; the frost-resistance of the silicate coating is 75 cycles. The developed facade cement-silicate coating can be applied on the surface of silicate-containing materials. The cost effectiveness of using the multifunctional protectivedecorative coating has been proved in comparison with similar compositions.
\end{abstract}

\section{Introduction}

At present, there is a growing interest in environmentally friendly building materials that have both increased durability and aesthetic properties [1-3]. One of the best materials having these properties is ceramic brick. Facing ceramic brick has a high durability, weather resistance, and decorative value and also functions as a construction and facing material.

Using a wide color palette, texture and shape of decorative facing ceramic bricks enhances the architectural treatment of residential and civil buildings. A number of enterprises manufacturing ceramic bricks improve the decorative properties of their products by means of volumetric pigmentation and glazing [or applying polymer-based fusion bonded

\footnotetext{
*Corresponding author: arina-shaybadullina@mail.ru
} 
paints (Fig. 1) [1]. The known methods for producing decorative brick have a number of disadvantages. In particular, the process of engobing and glazing facing brick is characterized with the complexity of technology and a relatively low durability of products and requires a lot of additional equipment [4].
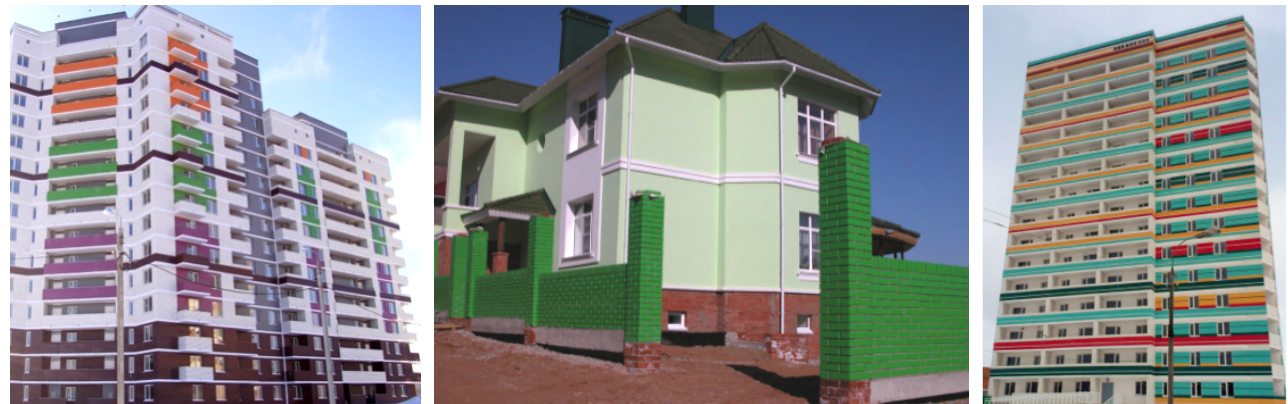

Fig. 1. General view of buildings with painted facing ceramic brick (Izhevsk, Russia).

The existing technology of painting brick surface with polymer fusion bonded paints has a significant disadvantage associated with the lack of vapor and gas permeability of the obtained coating. This disadvantage causes the damage of polymer coating of brick walls due to efflorescence that appears on the brick surface and separates the polymer coating from the base [5-7]. These processes are associated with the migration of salt solutions through the brickwork, which, as a rule, are present in the composition of a masonry solution (Fig. 2).

a)



b)



Fig. 2. General view of a polymer coating on the brickwork (a), a polymer coating separating from the brick surface due to efflorescence (b).

Thus, the development of a silicate coating with the improved physical and technical properties due to functional admixtures (multi-walled carbon nanotubes (MWCNTs), titanium dioxide [8], and expanded perlite [9] is a relevant task. Moreover, a facade coating, along with a high durability, acquires some additional properties, which provide special functions associated with the ability to absorb electromagnetic radiation, create the texture and color of the painted surface.

\section{Materials and methods of research}

This study focuses on the developed cement-silicate composition modified with ultra- and nanodispersed admixtures. The binder composition includes sodium silicate, Portland cement, an aqueous solution of sodium phosphate as a retarder, as well as ultrafine titanium dioxide, expanded perlite sand, and multi-walled carbon nanotubes suspension as modifiers of the structure. The required tint of the silicate composition was achieved by means of using 
a coloring paste of alkali-resistant pigments. The multi-walled carbon nanotubes suspension was first processed in a high-speed bead disperser and then treated with ultrasound in order to uniformly distribute multi-walled carbon nanotubes in the liquid phase and to avoid coagulation during storage.

The physical and technical characteristics of the developed silicate coating and the methods for their measurement meet the existing GOST and are given in Table 1. The relative viscosity of lacquer and paint is the time (in sec) of a continuous flow of a certain volume of the tested material through the calibration nozzle of viscometer. The viability of a lacquer and paint material is the maximum time during which this paint as separate components can be used after their mixing. The film resistance to the static effect of water is to determine the decorative and protective properties of coatings after their exposure to liquids during a given time. The adhesion is determined through the cross-cut test on the surface of the finished coating. Then the state of the coating is assessed visually according to the 6-point system (0 - no changes, 5 - more than $65 \%$ of the separating area). The vapor permeability and water absorption of the coating were determined similarly to the European standard ISO 7783:2011 and EN 1062-3:2008, respectively.

Table 1. Physical and technical characteristics of the facade silicate coating

\begin{tabular}{|l|l|l|}
\multicolumn{1}{|c|}{ Parameter } & \multicolumn{1}{|c|}{ Test method } & \multicolumn{1}{c|}{ Value } \\
\hline The viscosity measured with VZ-6 & GOST 8420-74 & 29 seconds \\
\hline $\begin{array}{l}\text { The viability (depending on the } \\
\text { retarder concentration) }\end{array}$ & GOST 27271-2014 & $110 \mathrm{~min}$ \\
\hline $\begin{array}{l}\text { The hydrogen index of the medium, } \\
\text { pH }\end{array}$ & GOST 52020-2003 & 12,33 \\
\hline $\begin{array}{l}\text { The film resistance to static effect of } \\
\text { water }\end{array}$ & GOST 9.403-80 & $8 \mathrm{~h}$ \\
\hline $\begin{array}{l}\text { The hiding power depending on the } \\
\text { pigment used }\end{array}$ & & $200-400 \mathrm{~g} / \mathrm{m}^{2}$ \\
\hline The adhesion by the cross-cut test & GOST 15140-78 & $1 \mathrm{point}$ \\
\hline $\begin{array}{l}\text { The vapor permeability of the paint } \\
\text { layer V }\end{array}$ & GOST 33355-2015 & $437 \mathrm{~g} / \mathrm{m}^{2} \cdot \mathrm{day}^{2}$ \\
\hline $\begin{array}{l}\text { The thickness of the air layer with the } \\
\text { equivalent vapor permeability, Sd }\end{array}$ & GOST 33355-2015 & $0.05 \mathrm{~m}$ \\
\hline The water absorption, W & GOST 33352-2015 & $0.80 \mathrm{~kg} / \mathrm{m}^{2} \cdot \mathrm{h}^{0.5}$ \\
\hline $\begin{array}{l}\text { The frost resistance of the silicate } \\
\text { coating }\end{array}$ & GOST 7025-91 & $75 \mathrm{cycles}$ \\
\hline
\end{tabular}

The shielding properties of carbon nanotubes are provided by their good conductivity. They have a low specific gravity and can be used for producing thin transparent films that preserve the appearance of a shielded object. This makes their usage as a protective coating more convenient [10]. 




Fig. 3. The shielding coefficient of the silicate coating depending on distance $\mathrm{L}$

The experiments studied the dependence of the shielding coefficient on the conditions of electromagnetic wave travel through a sample of material touching the test sample and the antennas located at a distance L (Fig. 3). The effective absorption capacity has a typical dependence and increases with the growing concentration multi-walled carbon nanotubes as a conductive filler whose content reached $7 \%$ of the mass of Portland cement. Adding carbon nanotubes into the composition of the studied silicate material leads to the formation of a binding silicate matrix and a dense, strong, and durable coating absorbing up to $38 \%$ of technogenic electromagnetic radiation affecting buildings and structures [11].

\section{Testing the developed cement-silicate coating}

The developed facade silicate coating, in contrast to a polymer coating, has the required porosity to ensure the vapor and gas permeability of brick walls. Moreover, unlike polymer coatings, the linear expansion coefficients of the developed silicate coating and ceramic brick have equal values, which eliminates the peeling of the silicate coating under alternating temperatures. The increased durability of the base silicate coating that the studied compositions are based on should also be noted. In particular, there are examples of buildings with the base silicate coating with a service life of more than 20 years (Fig. 4).

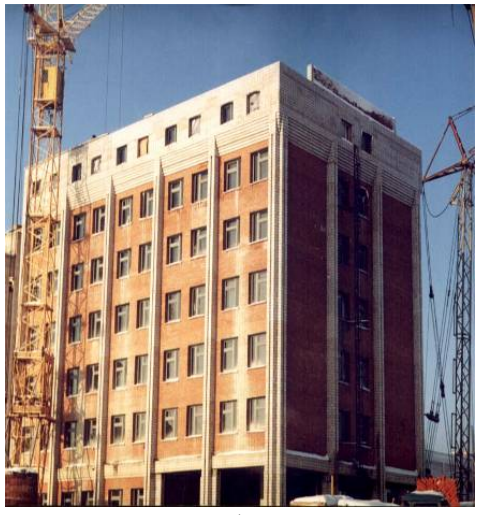

a)

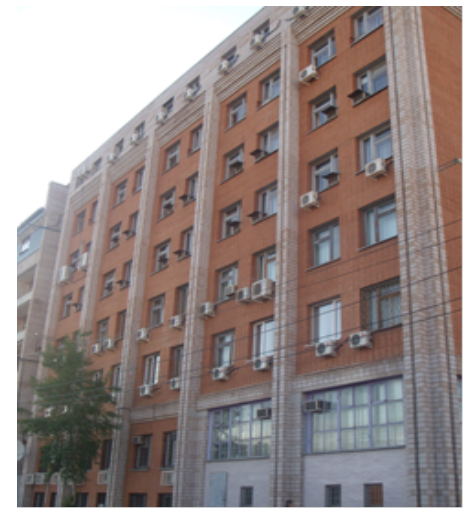

b)

Fig. 4. The building of Vneshterminalcomplex constructed in Izhevsk in 1996: a) - before painting in 1996, b) - while using the silicate coating in 2017.

The facade coating was tested in Altair, LTD, a factory producing ceramic brick (Izhevsk). Hollow ceramic bricks of M-100 were used as samples. The prepared silicate 
coating solution was applied with 1 layer using WESTER KP-10 air hopper gun (Fig. 5) under the air pressure created by FIAC EURO 25 compressor.

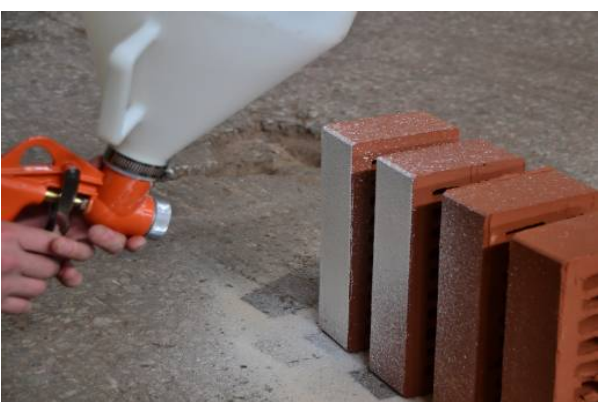

a)

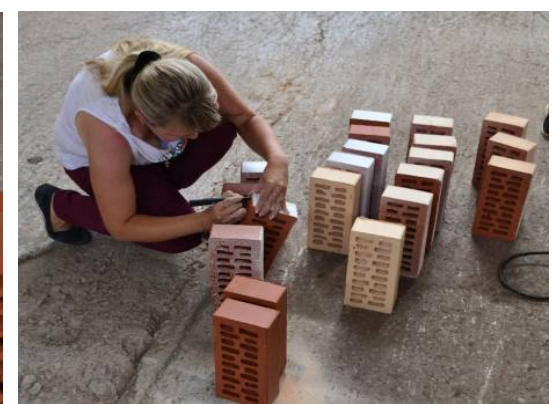

b)

Fig. 5. Applying the coating with a paint sprayer (a), marking the ceramic brick with the applied coating (b)

The following types of coatings were used in the experiment: 1 - a conventional composition without admixtures, 2 - a composition modified with a multi-walled carbon nanotubes dispersion. The pigments used was alkali-resistant coloring pastes produced by Novyy Dom, LTD (Izhevsk) (Fig.6).

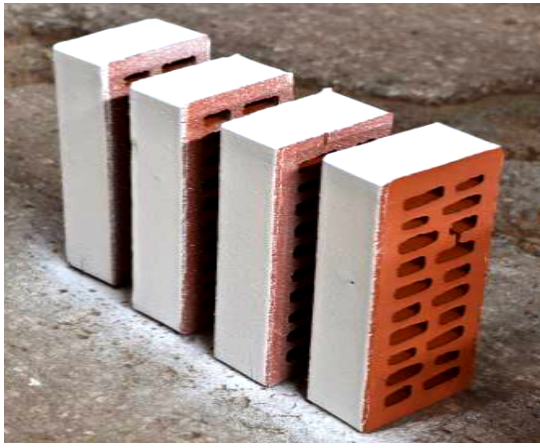

a)

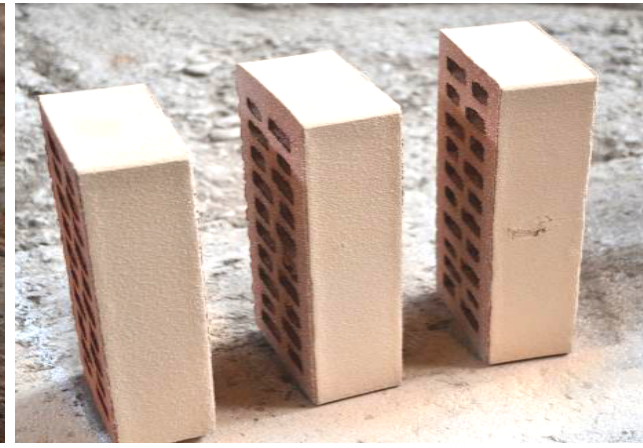

b)

Fig. 6. Ceramic brick coated with the decorative silicate composition: a) - white, b) - yellow

Expanded perlite had to be added due to the demand for textured coatings applied when using colored facing brick in residential buildings. High water resistance of expanded perlite in combination with the silicate coating of increased water resistance provides a textured coating on a facade surface. In this case, the pores in the structure of expanded perlite are blocked with a cement-silicate composition and at the same time act as a damper for water that crystallizes under negative temperature. This increases the frost resistance of the silicate coating. Thus, the peeling of the textured layer under alternating temperatures is avoided.

The studied compositions of the facade coating were also tested at the construction site during the erection of a residential house in Nizhnyaya street, d.12a (Izhevsk). To apply the silicate coating, fragments of brickwork with dimensions of $1000 \times 1000 \mathrm{~mm}$ and a thickness of $120 \mathrm{~mm}$ were built. In the process of applying, the compositions both with and without a MWCNTs dispersion were used. After being applied, the MWCNTs-modified coating had a more uniform and smoother surface in comparison with the conventional composition without nanotubes (Fig. 7). 


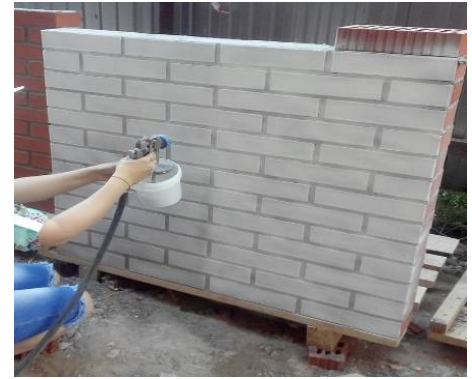

a)

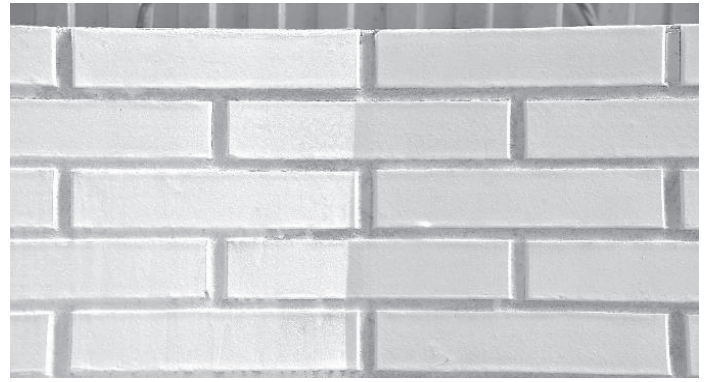

b)

Fig. 7. General view of the surface without pigment (a): b) - on the right - the coating composition without nanotubes, on the left - the coating with a MWCNTs dispersion

The developed facade silicate coating was used for painting brick walls of a stab-in boiler house in a residential building in Soyuznaya Street, 1, Izhevsk. The paint was applied in 3 layers on a dry base, which was previously cleaned from dust and dirt. The facades were painted at a temperature of $+15^{\circ} \mathrm{C}$. The composition of the finishing coating (3rd layer) had a MWCNTs dispersion. The primer layer was a thin layer of facade silicate coating without pigment (8b) providing the required adhesion for the follow-up application of the finishing coating. Each next layer of the pigmented facade silicate coating was applied on the surface after thorough drying (1-2 hours).

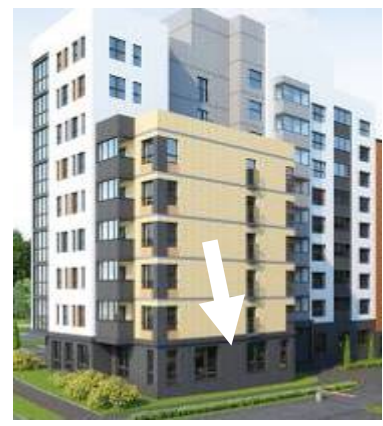

a)

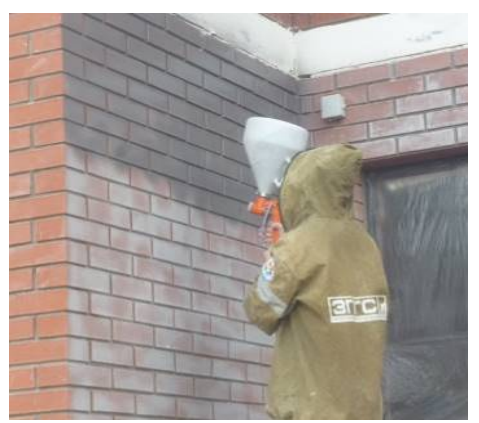

b)

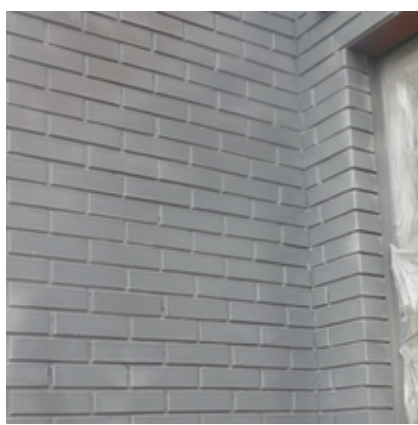

c)

Fig.8. The design color solution of facades of a residential building (the arrow shows the place of painting) (a); applying the finishing layer of the silicate coating with a spray gun (b); general view of the walls after painting (c).

Adding a MWCNTs dispersion into the composition of the applied silicate coating led to the improved technological properties of the coating. At the same time, the viscosity of the paintwork was reduced due to the structural modification of the components of the cementsilicate composition. The curing of the silicate coating modified with carbon nanotubes stimulates the structural formation of compositions with a denser structure reinforced with nanotubes. This provides the improved physical and technical properties and a shielding effect from technogenic electromagnetic radiation.

\section{Conclusion}

Applying the silicate coating on the surface of freshly burned ceramic bricks under industrial conditions has shown the possibility of replacing the existing technology of applying a polymer coating with the developed silicate composition. The advantages of the developed silicate coating are the absence of thermal processes associated with paint curing, the 
simplicity of applying by means of spray guns, the possibility of creating a textured layer and providing equal coefficients for linear expansion of the substrate and coating.

Testing the coating at the construction site confirmed the processability of its application and the high performance of painting work. The applied silicate coating confirmed the viability of the composition under environmental conditions when exposed to atmospheric precipitation.

\section{References}

1. S. K. Ghosh, G. Waghoo, A. Kalita, D. Balgude, K. R Kumar. Progress in Organic Coatings 73, 70-75 (2012)

2. L. X. Han, J. Han, F. M. Sun, Y. J Huo. Advanced Mater. Res.160-162, 880-885 (2011)

3. L. Kleerekoper, A. Dobbelsteen. van den, E. Ham. van den, T. Hordijk, C. Martin, Urban Climate 14, 290-300 (2015)

4. H. Li, L. Dong, Z. Jiang, X. Yang, Z. Yang. J. of Cleaner Production 133, 1017-1026 (2016)

5. G. Yakovlev, G. Pervushin, O. Kizievich, Y. Ginchickaya, P. Tajbahtina Stroitel'nye materialy 4, 69-71 (2016)

6. R.J. Flatt, J. Crystal Growth 242, 435-454 (2002)

7. H. Brocken, T. G. Nijland, Constr. Build. Mater. 18, 315-323 (2004)

8. L. Graziani, E. Quagliarini, M. D’Orazio, Constr. Build. Mater. 129, 116-124 (2016)

9. İ. B. Topçu, B. Işıkdăg, Build. Env. 42, 3540-3546 (2007)

10. B. Wanga, Z. Guoa, Y. Hana, T. Zhang, Constr. Build. Mater. 46, 98-103 (2013)

11. A. Shaybadullina, A. Politaeva, G. Yakovlev, G Pervushin, Adv. Mater. Res. 1122, 121$126(2015)$ 
